\title{
A rigorous derivation of the time-dependent Reynolds equation
}

\author{
John Fabricius ${ }^{\mathrm{a}, *}$, Yulia Koroleva ${ }^{\mathrm{a}, \mathrm{b}}$ and Peter Wall ${ }^{\mathrm{a}}$ \\ ${ }^{a}$ Department of Engineering Sciences and Mathematics, Luleå University of Technology, \\ SE-971 87 Luleå, Sweden \\ ${ }^{\mathrm{b}}$ Department of Differential Equations, Faculty of Mechanics and Mathematics, \\ Moscow Lomonosov State University, Moscow 119991, Russia
}

\begin{abstract}
We study the asymptotic behavior of solutions of the evolution Stokes equation in a thin three-dimensional domain bounded by two moving surfaces in the limit as the distance between the surfaces approaches zero. Using only a priori estimates and compactness it is rigorously verified that the limit velocity field and pressure are governed by the time-dependent Reynolds equation.
\end{abstract}

Keywords: lubrication theory, thin film, asymptotic behavior, evolution Stokes equation, time-dependent Reynolds equation

\section{Introduction}

In 1886 Osborne Reynolds [7] derived, on physical grounds, a two-dimensional equation describing the flow of a viscous fluid which is brought into motion by two rigid surfaces in close proximity. This equation, later known as the Reynolds equation, marks the foundation of lubrication theory and has since become the standard tool for computing the pressure distribution in various types of bearings. As Reynolds' lubrication equation is used in more general settings than the rather simple one in which it was originally derived, it is important to affirm the validity of the approximation from a theoretical point of view.

Rigorous lubrication theory is founded on a general fluid model, such as the full Navier-Stokes equation or intermediate models, and provides careful justifications for all the simplifications that lead to the Reynolds equation. Mathematically this means studying the asymptotic behavior of the flow in the limit as $\varepsilon$, a parameter that describes the relative gap between the surfaces, approaches zero. The limiting equations can be found by formally expanding velocity field, pressure and related quantities (notably the forces on the rigid surfaces) in a power series in $\varepsilon$, as shown in e.g. [4]. The asymptotic solution should also be compared to the "true" solution by studying convergence.

The asymptotic behavior of an incompressible viscous fluid in a thin domain has been studied in numerous papers. In 1983 Cimatti [3] considered the Stokes equation in a thin two-dimensional domain. As in Reynolds' original paper, it is assumed that the lower boundary is flat, moving at constant speed whereas the upper boundary is curved and at rest. These particular circumstances lead to a stationary problem. Cimatti then compares the solution of the Stokes equation, formulated in terms of a

\footnotetext{
*Corresponding author. E-mail: john.fabricius@ltu.se.
} 
stream function, to the flow corresponding to the one-dimensional Reynolds equation by estimating the $L^{2}$-norm of the difference. The generalization to three dimensions is due to Bayada and Chambat [1] whose approach is based on formal asymptotic expansion, energy estimates and compactness. Also in this study, the assumptions on the rigid surfaces are such that the resulting problem becomes stationary. Under additional hypotheses on the boundary data, convergence of velocity field and pressure is proved. A notable conclusion in [1] is that the proper boundary condition for the Reynolds equation is of Neumann type, although a Dirichlet condition is often used in reality for practical reasons. A more detailed review of the above cited works is found in the introduction of [9]. Let us finally mention that Marušić and Marušić-Paloka [6] introduced in 2000 a technique called "two-scale convergence for thin domains". As one of several applications, they obtain a degenerate Reynolds equation as the asymptotic limit of the stationary Navier-Stokes equation in a thin domain where the upper and lower boundaries meet at a sharp edge. Although the actual problem bears little resemblance to classical lubrication in that the boundaries are fixed and that the flow is driven by an external body force, the method is appealing thanks to its generality.

The present analysis deals with the asymptotic behavior of incompressible Stokes flow in a thin threedimensional domain bounded by two moving rigid surfaces. The assumptions regarding curvature and motion of the surfaces are sufficiently general to include most realistic applications and lead to a timedependent problem with a non-cylindrical space-time domain. This causes the main difficulty compared to the stationary case. In the limit as $\varepsilon \rightarrow 0$, we rigorously derive the time-dependent Reynolds equation and show how the limiting velocity field and pressure are governed by the Reynolds equation. The corresponding problem in two dimensions has been considered in [2], where the transition from the nonlinear Navier-Stokes equation to the Reynolds equation is proved. Due to well-known difficulties associated to estimating the inertial term in the three-dimensional case, see [2, Remark 4.1], we restrict our study to the linear Stokes equation. Our approach, entirely based on a priori estimates and compactness, follows that of Bayada et al. [2] but differs in some aspects. Notably, in regard to the derivation of estimates and the passage to the limit in the Stokes equation. The main improvement is that we are able to derive the Reynolds equation and deduce weak convergence of the velocity field without any bounds on the pressure. Let us also mention that the assumptions in [2] on the boundary data are unnecessarily restrictive, whereas we allow both surfaces to be curved and in "arbitrary" motion.

\section{Statement of the problem}

Let $\omega$ be an open bounded subset in $\mathbb{R}^{2}$, with sufficiently smooth boundary. Let $h^{+}, h^{-} \in C^{2}\left(\mathbb{R}^{2} \times\right.$ $[0, T])$ such that

$$
h_{\min } \leqslant h=h^{+}-h^{-} \leqslant h_{\max },
$$

where $T>0$ is given and $h_{\min }, h_{\max }$ are positive constants. For $t \in[0, T]$ define the thin film

$$
\Omega_{\varepsilon}(t)=\left\{x \in \mathbb{R}^{3}: x^{\prime}=\left(x_{1}, x_{2}\right) \in \omega, \varepsilon h^{-}\left(x^{\prime}, t\right)<x_{3}<\varepsilon h^{+}\left(x^{\prime}, t\right)\right\} .
$$

The boundary $\partial \Omega_{\varepsilon}(t)$ can be split into three disjoint parts:

$$
\partial \Omega_{\varepsilon}(t)=\Sigma_{\varepsilon}^{-}(t) \cup \Sigma_{\varepsilon}^{+}(t) \cup \Sigma_{\varepsilon}^{w}(t),
$$


where

$$
\begin{aligned}
& \Sigma_{\varepsilon}^{ \pm}(t)=\left\{x \in \mathbb{R}^{3}: x^{\prime}=\left(x_{1}, x_{2}\right) \in \omega, x_{3}=\varepsilon h^{ \pm}\left(x^{\prime}, t\right)\right\}, \\
& \Sigma_{\varepsilon}^{w}(t)=\left\{x \in \mathbb{R}^{3}: x^{\prime}=\left(x_{1}, x_{2}\right) \in \partial \omega, \varepsilon h^{-}\left(x^{\prime}, t\right) \leqslant x_{3} \leqslant \varepsilon h^{+}\left(x^{\prime}, t\right)\right\} .
\end{aligned}
$$

Furthermore, for any $t \in[0, T]$ set

$$
\Omega_{\varepsilon t}=\bigcup_{0<\tau \leqslant t} \Omega_{\varepsilon}(\tau) \times\{\tau\}, \quad \Sigma_{\varepsilon t}^{ \pm}=\bigcup_{0<\tau \leqslant t} \Sigma_{\varepsilon}^{ \pm}(\tau) \times\{\tau\}, \quad \Sigma_{\varepsilon t}^{w}=\bigcup_{0<\tau \leqslant t} \Sigma_{\varepsilon}^{w}(\tau) \times\{\tau\} .
$$

We consider the incompressible time-dependent Stokes equation in $\Omega_{\varepsilon T}$, i.e.

$$
\begin{aligned}
& D_{t} U^{\varepsilon}-\nu \Delta U^{\varepsilon}+\nabla P^{\varepsilon}=0 \text { in } \Omega_{\varepsilon T}, \\
& \operatorname{div} U^{\varepsilon}=0 \text { in } \Omega_{\varepsilon T},
\end{aligned}
$$

where $D_{t}=\partial / \partial t, \nu$ (kinematic viscosity) is a positive constant, $U^{\varepsilon}$ (velocity field) and $P^{\varepsilon}$ (pressure) are unknowns, with initial-boundary values

$$
\begin{aligned}
& U^{\varepsilon}=\left(v_{1}^{ \pm}, v_{2}^{ \pm}, \varepsilon v_{3}^{ \pm}\right) \quad \text { on } \Sigma_{\varepsilon T}^{ \pm} \text {(no-slip condition), } \\
& U^{\varepsilon}=g^{\varepsilon} \quad \text { on } \Sigma_{\varepsilon T}^{w}, \\
& U^{\varepsilon}=U_{0}^{\varepsilon} \quad \text { on } \Omega_{\varepsilon}(0),
\end{aligned}
$$

where $g^{\varepsilon}$ and $U_{0}^{\varepsilon}$ are described below. Following [2], it is assumed that $g^{\varepsilon} \in H^{1 / 2}\left(\Sigma_{\varepsilon T}^{w} ; \mathbb{R}^{3}\right)$ and $U_{0}^{\varepsilon} \in$ $H^{1}\left(\Omega_{\varepsilon}(0) ; \mathbb{R}^{3}\right)$ are of the form

$$
\begin{aligned}
& g^{\varepsilon}(x, t)=E \hat{g}\left(E^{-1} x, t\right), \\
& U_{0}^{\varepsilon}(x)=E \hat{U}_{0}\left(E^{-1} x\right),
\end{aligned}
$$

for all $\varepsilon>0$, where $\hat{g}$ and $\hat{U}_{0}$ are independent of $\varepsilon$ (see [2] for more details) and $E$ is the matrix

$$
E(\varepsilon)=\left(\begin{array}{lll}
1 & 0 & 0 \\
0 & 1 & 0 \\
0 & 0 & \varepsilon
\end{array}\right)
$$

and that for a.e. $t \in(0, T)$ the following compatibility condition is satisfied:

$$
\int_{\omega} D_{t} h\left(x^{\prime}, t\right) \mathrm{d} x^{\prime}+\int_{\partial \omega}\left(\int_{h^{-}\left(x^{\prime}, t\right)}^{h^{+}\left(x^{\prime}, t\right)} \widehat{g}\left(x^{\prime}, z, t\right) \mathrm{d} z\right) \cdot \hat{n} \mathrm{~d} S\left(x^{\prime}\right)=0
$$

where $\hat{n}$ denotes the outward unit normal. Assumptions on $v_{i}^{ \pm}$and $U_{0}^{\varepsilon}$ are

$$
\begin{aligned}
& v^{ \pm}=\left(v_{1}^{ \pm}, v_{2}^{ \pm}\right) \in C\left(\bar{\omega} \times[0, T] ; \mathbb{R}^{2}\right) \\
& v_{3}^{ \pm}=D_{t} h^{ \pm}+v_{1}^{ \pm} D_{1} h^{ \pm}+v_{2}^{ \pm} D_{2} h^{ \pm}
\end{aligned}
$$




$$
\begin{aligned}
& U_{0}^{\varepsilon}= \begin{cases}\left(v_{1}^{ \pm}, v_{2}^{ \pm}, \varepsilon v_{3}^{ \pm}\right) & \text {on } \Sigma_{\varepsilon}^{ \pm}(0), \\
g^{\varepsilon} & \text { on } \Sigma_{\varepsilon}^{w}(0),\end{cases} \\
& \operatorname{div} U_{0}^{\varepsilon}=0 \quad \text { in } \Omega_{\varepsilon}(0), \\
& \int_{\omega} \int_{h^{-}\left(x^{\prime}, 0\right)}^{h^{+}\left(x^{\prime}, 0\right)}\left|\hat{U}_{0}\left(x^{\prime}, z\right)\right|^{2} \mathrm{~d} z \mathrm{~d} x^{\prime}<\infty .
\end{aligned}
$$

Here $D_{i}(i=1,2,3)$ denotes the $i$ th partial derivative. Moreover, if $u: \mathbb{R}^{3} \rightarrow \mathbb{R}^{3}, D u$ denotes the $3 \times 3$ matrix with $D_{j} u_{i}$ in row $i$ column $j$. In this regard, if $A=\left\{a_{i j}\right\}$ and $B=\left\{b_{i j}\right\}$ are matrices of equal dimensions their Frobenius product is defined as $A: B=\sum_{i, j} a_{i j} b_{i j}$, which induces a Euclidean norm $|A|=\sqrt{A: A}$.

Remark 2.1. The typical example of rigid body motion in lubrication is

$$
\begin{aligned}
& v_{1}^{ \pm}(x, t)=-\alpha^{ \pm}(t) x_{2}+\beta_{1}^{ \pm}(t), \\
& v_{2}^{ \pm}(x, t)=\alpha^{ \pm}(t) x_{1}+\beta_{2}^{ \pm}(t), \\
& v_{3}^{ \pm}(x, t)=0,
\end{aligned}
$$

where $\left(0,0, \alpha^{ \pm}\right)$and $\left(\beta_{1}^{ \pm}, \beta_{2}^{ \pm}, 0\right)$ are angular and translational velocity vectors respectively. However, (2.11)-(2.12) do not require that the bounding surfaces be rigid. In fact, $v_{1}^{ \pm}, v_{2}^{ \pm}$are arbitrary continuous functions, whereas $v_{3}^{ \pm}$and $h^{ \pm}$must be compatible through (2.11). Regarding certain conditions on the geometry and motion of $\Sigma_{\varepsilon}^{ \pm}(t)$ for such $h^{ \pm}$to exist we refer to the introduction to [4].

We introduce the new unknown function $\hat{u}^{\varepsilon}=U^{\varepsilon}-\hat{\mathcal{U}}^{\varepsilon}$, where $\hat{\mathcal{U}}^{\varepsilon}$ is chosen so that $\hat{u}^{\varepsilon}$ satisfies homogeneous boundary condition. It is assumed that there exists a measurable vector field $\hat{\mathcal{U}}$ such that

$$
\begin{aligned}
& \hat{\mathcal{U}}^{\varepsilon}(x, t)=E \hat{\mathcal{U}}\left(E^{-1} x, t\right), \\
& \operatorname{div} \hat{\mathcal{U}}^{\varepsilon}=0 \quad \text { in } \Omega_{\varepsilon T}, \\
& \hat{\mathcal{U}}^{\varepsilon}=U^{\varepsilon} \text { on } \Sigma_{\varepsilon T}^{ \pm} \cup \Sigma_{\varepsilon T}^{w}, \\
& \int_{0}^{T} \int_{\omega} \int_{h^{-}\left(x^{\prime}, t\right)}^{h^{+}\left(x^{\prime}, t\right)}\left|\hat{\mathcal{U}}\left(x^{\prime}, z, t\right)\right|^{2}+\left|D_{t} \hat{\mathcal{U}}\left(x^{\prime}, z, t\right)\right|^{2}+\left|D \hat{\mathcal{U}}\left(x^{\prime}, z, t\right)\right|^{2} \mathrm{~d} z \mathrm{~d} x^{\prime} \mathrm{d} t<\infty .
\end{aligned}
$$

Thus we obtain the following equation for $\hat{u}^{\varepsilon}$

$$
\begin{aligned}
& D_{t} \hat{u}^{\varepsilon}-\nu \Delta \hat{u}^{\varepsilon}+\nabla P^{\varepsilon}=f^{\varepsilon} \quad \text { in } \Omega_{\varepsilon T}, \\
& \operatorname{div} \hat{u}^{\varepsilon}=0 \quad \text { in } \Omega_{\varepsilon T}, \\
& \hat{u}^{\varepsilon}=0 \quad \text { on } \Sigma_{\varepsilon T}^{ \pm} \cup \Sigma_{\varepsilon T}^{w}, \\
& \hat{u}^{\varepsilon}(x, 0)=\hat{u}_{0}^{\varepsilon} \quad \text { in } \Omega_{\varepsilon}(0),
\end{aligned}
$$

where

$$
f^{\varepsilon}=-D_{t} \hat{\mathcal{U}}^{\varepsilon}+\nu \Delta \hat{\mathcal{U}}^{\varepsilon}
$$


and $\hat{u}_{0}^{\varepsilon}(x)=U_{0}^{\varepsilon}(x)-\hat{\mathcal{U}}^{\varepsilon}(x, 0)$. Let $\varepsilon_{0}>0$ be given. Then it follows from (2.14) and (2.18) that there exists a constant $K$ (which depends on $\varepsilon_{0}$ ) such that

$$
\int_{\Omega_{\varepsilon}(0)}\left|\hat{u}_{0}^{\varepsilon}\right|^{2} \mathrm{~d} x \leqslant \varepsilon K
$$

for all $0<\varepsilon \leqslant \varepsilon_{0}$.

As a first step towards a definition of weak solution for this problem we define the bilinear (in $u$ and $v$ ) form

$$
\hat{\mathcal{S}}^{\varepsilon}(u, v, t)=\int_{\Omega_{\varepsilon}(t)} D_{t} u \cdot v+\nu D u: D v \mathrm{~d} x
$$

Then, for each $t \in(0, T]$, a smooth solution $\hat{u}^{\varepsilon}$ of (2.19)-(2.22) must satisfy the identity

$$
\hat{\mathcal{S}}^{\varepsilon}\left(\hat{u}^{\varepsilon}, v, t\right)=-\hat{\mathcal{S}}^{\varepsilon}\left(\hat{\mathcal{U}}^{\varepsilon}, v, t\right)
$$

for all smooth $v: \Omega_{\varepsilon}(t) \rightarrow \mathbb{R}^{3}$ with compact support such that $\operatorname{div} v=0$.

The goal of this paper is to study the asymptotic behavior of $\hat{u}^{\varepsilon}$ as $\varepsilon \rightarrow 0$. The main result is formulated in Theorem 5.1 which states that the limit flow is governed by the Reynolds equation (5.20).

\section{Casting the problem in a fixed domain}

The above problem, formulated using the natural choice of coordinates, is complicated to analyze due to the time-dependent space domain. To circumvent this, the domain $\Omega_{\varepsilon}(t)$ is transformed into $\Omega=\omega \times(0,1)$, which depends neither on $\varepsilon$ nor $t$, by a change of variables (see Fig. 1). A weak solution for the considered problem may then be defined in terms of $\Omega$.

To this end let $\psi^{\varepsilon}(\cdot, t): \Omega \rightarrow \Omega_{\varepsilon}(t)$ be defined by

$$
\psi^{\varepsilon}(\xi, t)=\left(\xi^{\prime},\left(1-\xi_{3}\right) \varepsilon h^{-}\left(\xi^{\prime}, t\right)+\xi_{3} \varepsilon h^{+}\left(\xi^{\prime}, t\right)\right),
$$

where $\xi^{\prime}=\left(\xi_{1}, \xi_{2}\right)$ is a point in $\omega$. The following notation convention is applied throughout the paper:

A point $x \in \Omega_{\varepsilon}(t)$ and a point $\xi \in \Omega$ are related through $x=\psi^{\varepsilon}(\xi, t)$ or equivalently $\xi=\phi^{\varepsilon}(x, t)$, where $\phi^{\varepsilon}(\cdot, t): \Omega_{\varepsilon}(t) \rightarrow \Omega$ is the inverse of $\psi^{\varepsilon}(\cdot, t)$.

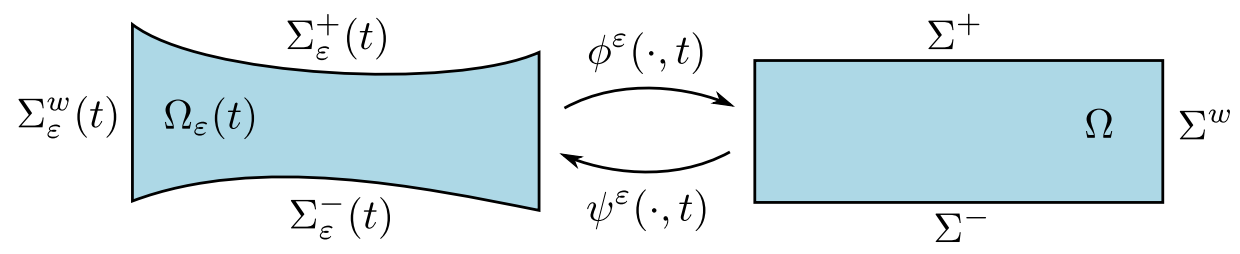

Fig. 1. Cross sections of the domains $\Omega_{\varepsilon}(t)$ and $\Omega$. (Colors are visible in the online version of the article; http://dx.doi.org/ 10.3233/ASY-131165.) 
We have

$$
\partial \Omega=\Sigma^{-} \cup \Sigma^{+} \cup \Sigma^{w},
$$

where

$$
\begin{aligned}
& \Sigma^{-}=\left\{\xi \in \mathbb{R}^{3}: \xi^{\prime} \in \omega \text { and } \xi_{3}=0\right\}, \\
& \Sigma^{+}=\left\{\xi \in \mathbb{R}^{3}: \xi^{\prime} \in \omega \text { and } \xi_{3}=1\right\}, \\
& \Sigma^{w}=\left\{\xi \in \mathbb{R}^{3}: \xi^{\prime} \in \partial \omega \text { and } 0 \leqslant \xi_{3} \leqslant 1\right\}
\end{aligned}
$$

and set $\Omega_{T}=\Omega \times(0, T), \omega_{T}=\omega \times(0, T)$.

Furthermore, let

$$
\begin{aligned}
A^{\varepsilon}(\xi, t) & =\left\{D_{j} \psi_{i}^{\varepsilon}(\xi, t)\right\} \\
& =\left(\begin{array}{ccc}
1 & 0 & 0 \\
0 & 1 & 0 \\
\varepsilon\left(1-\xi_{3}\right) D_{1} h^{-}+\varepsilon \xi_{3} D_{1} h^{+} & \varepsilon\left(1-\xi_{3}\right) D_{2} h^{-}+\varepsilon \xi_{3} D_{2} h^{+} & \varepsilon h
\end{array}\right)
\end{aligned}
$$

and define $B^{\varepsilon}$ as the inverse matrix of $A^{\varepsilon}$, i.e.

$$
B^{\varepsilon}(\xi, t)=\left(\begin{array}{ccc}
1 & 0 & 0 \\
0 & 1 & 0 \\
-\frac{\left(1-\xi_{3}\right) D_{1} h^{-}+\xi_{3} D_{1} h^{+}}{h} & -\frac{\left(1-\xi_{3}\right) D_{2} h^{-}+\xi_{3} D_{2} h^{+}}{h} & \frac{1}{\varepsilon h}
\end{array}\right) .
$$

Note that $\operatorname{det} A^{\varepsilon}=\varepsilon h$. We sometimes write $A^{\varepsilon}=E A$ and $B^{\varepsilon}=B E^{-1}$ where

$$
A=\left(\begin{array}{lll}
1 & 0 & 0 \\
0 & 1 & 0 \\
a & b & h
\end{array}\right), \quad B=A^{-1}=\left(\begin{array}{ccc}
1 & 0 & 0 \\
0 & 1 & 0 \\
-a / h & -b / h & 1 / h
\end{array}\right),
$$

$a=\left(1-\xi_{3}\right) D_{1} h^{-}+\xi_{3} D_{1} h^{+}$and $b=\left(1-\xi_{3}\right) D_{2} h^{-}+\xi_{3} D_{2} h^{+}$.

Let us now see how $\hat{\mathcal{S}}^{\varepsilon}$, defined by (2.25), transforms under this change of variables.

Lemma 3.1. Let $\mathcal{S}^{\varepsilon}$ be the bilinear form

$$
\mathcal{S}^{\varepsilon}(u, v, t)=\int_{\Omega}\left(h D_{t} u-c D_{3} u\right) \cdot v+\nu h D u B^{\varepsilon}: D v B^{\varepsilon} \mathrm{d} \xi,
$$

where $c=\left(1-\xi_{3}\right) D_{t} h^{-}+\xi_{3} D_{t} h^{+}$, and let $F^{\varepsilon}$ be the linear functional

$$
\left\langle F^{\varepsilon}(t), v\right\rangle=-\varepsilon^{-1} \hat{\mathcal{S}}^{\varepsilon}\left(\hat{\mathcal{U}}^{\varepsilon}, v \circ \phi^{\varepsilon}, t\right) .
$$

Then a smooth field $\hat{u}^{\varepsilon}: \Omega_{\varepsilon}(t) \rightarrow \mathbb{R}^{3}$ satisfies

$$
\hat{\mathcal{S}}^{\varepsilon}\left(\hat{u}^{\varepsilon}, v, t\right)=-\hat{\mathcal{S}}^{\varepsilon}\left(\hat{\mathcal{U}}^{\varepsilon}, v, t\right)
$$


for all smooth $v: \Omega_{\varepsilon}(t) \rightarrow \mathbb{R}^{3}$ with compact support, if and only if $u^{\varepsilon}=\hat{u}^{\varepsilon} \circ \psi^{\varepsilon}$, i.e.

$$
u^{\varepsilon}(\xi, t)=\hat{u}^{\varepsilon}\left(\psi^{\varepsilon}(\xi, t), t\right),
$$

satisfies

$$
\mathcal{S}^{\varepsilon}\left(u^{\varepsilon}, v, t\right)=\left\langle F^{\varepsilon}(t), v\right\rangle
$$

for all smooth $v: \Omega \rightarrow \mathbb{R}^{3}$ with compact support. Moreover

$\operatorname{div} \hat{u}^{\varepsilon}=0 \quad$ in $\Omega_{\varepsilon}(t)$

if and only if

$$
\operatorname{div}\left(h B^{\varepsilon} u^{\varepsilon}\right)=0 \quad \text { in } \Omega .
$$

Proof. By the chain rule we have

$$
\begin{aligned}
& D \phi^{\varepsilon}(x, t)=D \psi^{\varepsilon}(\xi, t)^{-1}=B^{\varepsilon}(\xi, t), \\
& D_{t} \phi_{3}^{\varepsilon}(x, t)=-\frac{D_{t} \psi_{3}^{\varepsilon}(\xi, t)}{D_{3} \psi_{3}^{\varepsilon}(\xi, t)}=-\frac{c}{h} .
\end{aligned}
$$

Thus

$$
\begin{aligned}
\hat{\mathcal{S}}^{\varepsilon}\left(u \circ \phi^{\varepsilon}, v \circ \phi^{\varepsilon}, t\right)= & \int_{\Omega_{\varepsilon}(t)}\left(D_{t} u(\xi, t)+D_{t} \phi_{3}^{\varepsilon}(x, t) D_{3} u(\xi, t)\right) \cdot v(\xi) \\
& \quad+\nu D u(\xi, t) D \phi^{\varepsilon}(x, t): D v(\xi, t) D \phi^{\varepsilon}(x, t) \mathrm{d} x \\
= & \int_{\Omega}\left(\left(D_{t} u-\frac{c}{h} D_{3} u\right) \cdot v+\nu D u B^{\varepsilon}: D v B^{\varepsilon}\right)\left|\operatorname{det} A^{\varepsilon}\right| \mathrm{d} \xi \\
= & \varepsilon \int_{\Omega}\left(h D_{t} u-\varepsilon c D_{3} u\right) \cdot v+\nu h D u B^{\varepsilon}: D v B^{\varepsilon} \mathrm{d} \xi=\varepsilon \mathcal{S}^{\varepsilon}(u, v, t) .
\end{aligned}
$$

Hence

$$
\mathcal{S}^{\varepsilon}(u, v, t)=\varepsilon^{-1} \hat{\mathcal{S}}^{\varepsilon}\left(u \circ \phi^{\varepsilon}, v \circ \phi^{\varepsilon}, t\right)
$$

or equivalently

$$
\hat{\mathcal{S}}^{\varepsilon}(u, v, t)=\varepsilon \mathcal{S}^{\varepsilon}\left(u \circ \psi^{\varepsilon}, v \circ \psi^{\varepsilon}, t\right) .
$$

Let us now show that $\operatorname{div}\left(u \circ \phi^{\varepsilon}\right)=0$ in $\Omega_{\varepsilon}(t)$ is equivalent to $\operatorname{div}\left(h B^{\varepsilon} u\right)=0$ in $\Omega$. Indeed

$$
\begin{aligned}
\int_{\Omega_{\varepsilon}(t)} u \circ \phi^{\varepsilon} \cdot \nabla\left(v \circ \phi^{\varepsilon}\right) \mathrm{d} x & =\int_{\Omega_{\varepsilon}(t)} D \phi^{\varepsilon}(x) u(\xi) \cdot \nabla v(\xi) \mathrm{d} x \\
& =\int_{\Omega} B^{\varepsilon} u \cdot \nabla v\left|\operatorname{det} A^{\varepsilon}\right| \mathrm{d} \xi=\int_{\Omega} \varepsilon h B^{\varepsilon} u \cdot \nabla v \mathrm{~d} \xi
\end{aligned}
$$

for all smooth $v: \Omega \rightarrow \mathbb{R}$ with compact support. 
The preceding lemma motivates the following definition of generalized solution which is formally obtained by replacing $v$ in (3.5) with $A^{\varepsilon} v / h$, where $\operatorname{div} v=0$.

Definition 1. Let

$$
\begin{aligned}
& H=\left\{u \in L^{2}\left(\Omega ; \mathbb{R}^{3}\right): \operatorname{div} u=0, u \cdot \hat{n}=0 \text { on } \partial \Omega\right\}, \\
& V=\left\{u \in H_{0}^{1}\left(\Omega ; \mathbb{R}^{3}\right): \operatorname{div} u=0 \text { in } \Omega\right\}
\end{aligned}
$$

and let $V^{\prime}$ denote the dual of $V$. We say that $\hat{u}^{\varepsilon}=u^{\varepsilon} \circ \phi^{\varepsilon}$ is a weak solution of (2.19)-(2.23) if $u^{\varepsilon}$ satisfies $h B^{\varepsilon} u^{\varepsilon} \in L^{2}(0, T ; V) \cap L^{\infty}(0, T ; H), D_{t}\left(\left(A^{\varepsilon}\right)^{T} u^{\varepsilon}\right) \in L^{2}\left(0, T ; V^{\prime}\right)$ and

$$
\begin{aligned}
& \frac{\mathrm{d}}{\mathrm{d} t} \int_{\Omega} u^{\varepsilon} \cdot A^{\varepsilon} v \mathrm{~d} \xi+\int_{\Omega}-u^{\varepsilon} \cdot D_{t} A^{\varepsilon} v-\frac{c}{h} D_{3} u^{\varepsilon} \cdot A^{\varepsilon} v+\nu h D u^{\varepsilon} B^{\varepsilon}: D\left(A^{\varepsilon} v / h\right) B^{\varepsilon} \mathrm{d} \xi \\
& \quad=\left\langle F^{\varepsilon}(t), A^{\varepsilon} v / h\right\rangle
\end{aligned}
$$

for all a.e. $t \in(0, T)$ and all $v \in V$ and satisfies

$$
u^{\varepsilon}(\xi, 0)=u_{0}^{\varepsilon}(\xi), \quad \xi \in \Omega,
$$

where $u_{0}^{\varepsilon}=\hat{u}_{0}^{\varepsilon} \circ \psi^{\varepsilon}$.

\section{Existence and uniqueness}

Thus, the idea is to prove existence and uniqueness of $u^{\varepsilon}$ and then define $\hat{u}^{\varepsilon}$ as $u^{\varepsilon} \circ \phi^{\varepsilon}$. The following result holds.

Theorem 4.1. The boundary-value problem (3.6) has a unique solution $u^{\varepsilon}$ for each $\varepsilon>0$. Moreover, there exists a constant $K$ (which depends on $\varepsilon_{0}$ ) such that

$$
\begin{aligned}
& \left\|u^{\varepsilon}\right\|_{\Omega_{T}}+\left\|D_{3} u^{\varepsilon}\right\|_{\Omega_{T}} \leqslant K, \\
& \sup _{0 \leqslant t \leqslant T}\left\|u^{\varepsilon}\right\|_{\Omega}+\left\|D_{1} u^{\varepsilon}\right\|_{\Omega_{T}}+\left\|D_{2} u^{\varepsilon}\right\|_{\Omega_{T}} \leqslant \varepsilon^{-1} K
\end{aligned}
$$

for all $0<\varepsilon \leqslant \varepsilon_{0}$.

To prove Theorem 4.1 we need some preliminary estimates. The construction of $u^{\varepsilon}$, defined by (3.6), is standard and relies on the so called Galerkin method. For simplicity we denote the norm in $L^{2}\left(Q ; \mathbb{R}^{k}\right)$ as $\|\cdot\|_{Q}$, where $Q$ is an arbitrary open set and $k=1,3,3 \times 3$ is clear from the context. The various constants introduced in the derivations are denoted by $K_{1}, K_{2}, \ldots$

Lemma 4.1. Let $B^{\varepsilon}$ and $E$ be given by (3.2) and (2.8) respectively. For each $\varepsilon_{0}>0$ there exist positive constants $\lambda^{-}, \lambda^{+}$such that

$$
\lambda^{-}\left|X E^{-1}\right|^{2} \leqslant\left|X B^{\varepsilon}\right|^{2} \leqslant \lambda^{+}\left|X E^{-1}\right|^{2}
$$

for all real $3 \times 3$ matrices $X$, for all $0<\varepsilon \leqslant \varepsilon_{0}$. 
Proof. Set $Y=\left(X E^{-1}\right)^{T}$, then

$$
\left|X B^{\varepsilon}\right|^{2}=\left|\left(E B E^{-1}\right)^{T} Y\right|^{2}=\sum_{i}\left|\left(E B E^{-1}\right)^{T} y_{i}\right|^{2}
$$

where $y_{i}$ denotes the $i$ th column vector of $Y$. Some elementary calculations show that any eigenvalue $\lambda=\lambda(\varepsilon)$ of the quadratic form

$$
Q^{\varepsilon}(y)=\left|\left(E B E^{-1}\right)^{T} y\right|^{2}, \quad y \in \mathbb{R}^{3}
$$

satisfies

$$
\frac{1}{1+h^{2}+\varepsilon^{2}\left(a^{2}+b^{2}\right)}<\lambda<1+\frac{1+\varepsilon^{2}\left(a^{2}+b^{2}\right)}{h^{2}} .
$$

Thus there exist positive constants $\lambda^{ \pm}$which depend on $\varepsilon_{0}, h^{+}$and $h^{-}$such that $\lambda^{-} \leqslant \lambda \leqslant \lambda^{+}$for all $\varepsilon \in\left[0, \varepsilon_{0}\right]$. Hence

$$
\lambda^{-}|Y|^{2} \leqslant\left|\left(E B E^{-1}\right)^{T} Y\right|^{2} \leqslant \lambda^{+}|Y|^{2}
$$

for all matrices $Y$ which implies (4.3).

Lemma 4.2. For each $\varepsilon_{0}>0$ there exists a constant $\lambda^{+}$such that

$$
\begin{aligned}
\left|\left\langle F^{\varepsilon}(t), v\right\rangle\right| \leqslant & \left(\int_{\omega} \int_{h^{-}}^{h^{+}}\left|E D_{t} \hat{\mathcal{U}}\right|^{2} \mathrm{~d} z \mathrm{~d} x^{\prime}\right)^{1 / 2}\left(\int_{\Omega}|v|^{2} h \mathrm{~d} \xi\right)^{1 / 2} \\
& +\nu \sqrt{\lambda^{+}}\left(\int_{\omega} \int_{h^{-}}^{h^{+}}\left|E D \hat{\mathcal{U}} E^{-1}\right|^{2} \mathrm{~d} z \mathrm{~d} x^{\prime}\right)^{1 / 2}\left(\int_{\Omega}\left|D v E^{-1}\right|^{2} h \mathrm{~d} \xi\right)^{1 / 2}
\end{aligned}
$$

for all $v \in H_{0}^{1}\left(\Omega ; \mathbb{R}^{3}\right)$, for all $0<\varepsilon \leqslant \varepsilon_{0}$.

Proof. From the definitions of $F^{\varepsilon}$ and $\hat{\mathcal{U}}^{\varepsilon}$

$$
\left\langle F^{\varepsilon}(t), v\right\rangle=-\varepsilon^{-1} \int_{\Omega_{\varepsilon}(t)} E D_{t} \hat{\mathcal{U}}\left(E^{-1} x, t\right) \cdot\left(v \circ \phi^{\varepsilon}\right)+\nu E D \hat{\mathcal{U}}\left(E^{-1} x, t\right) E^{-1}: D\left(v \circ \phi^{\varepsilon}\right) \mathrm{d} x .
$$

Thus, using Lemma 4.1,

$$
\begin{aligned}
\left|\left\langle F^{\varepsilon}(t), v\right\rangle\right| \leqslant & \varepsilon^{-1}\left(\int_{\omega} \int_{h^{-}}^{h^{+}}\left|E D_{t} \hat{\mathcal{U}}\right|^{2} \varepsilon \mathrm{d} z \mathrm{~d} x^{\prime}\right)^{1 / 2}\left(\int_{\Omega}|v|^{2} \varepsilon h \mathrm{~d} \xi\right)^{1 / 2} \\
& +\varepsilon^{-1} \nu\left(\int_{\omega} \int_{h^{-}}^{h^{+}}\left|E D \hat{\mathcal{U}} E^{-1}\right|^{2} \varepsilon \mathrm{d} z \mathrm{~d} x^{\prime}\right)^{1 / 2}\left(\int_{\Omega}\left|D v B^{\varepsilon}\right|^{2} \varepsilon h \mathrm{~d} \xi\right)^{1 / 2}
\end{aligned}
$$




$$
\begin{aligned}
\leqslant & \left(\int_{\omega} \int_{h^{-}}^{h^{+}}\left|E D_{t} \hat{\mathcal{U}}\right|^{2} \mathrm{~d} z \mathrm{~d} x^{\prime}\right)^{1 / 2}\left(\int_{\Omega}|v|^{2} h \mathrm{~d} \xi\right)^{1 / 2} \\
& +\nu \sqrt{\lambda^{+}}\left(\int_{\omega} \int_{h^{-}}^{h^{+}}\left|E D \hat{\mathcal{U}} E^{-1}\right|^{2} \mathrm{~d} z \mathrm{~d} x^{\prime}\right)^{1 / 2}\left(\int_{\Omega}\left|D v E^{-1}\right|^{2} h \mathrm{~d} \xi\right)^{1 / 2} .
\end{aligned}
$$

Proof of Theorem 4.1. We shall construct $u^{\varepsilon}$ as a Galerkin approximation. For this we choose an orthonormal basis $\left\{u^{n}\right\}_{n=1}^{\infty}$ in $H$ that is dense in the space $V$, e.g. the solutions of the eigenvalue problem

$$
\begin{aligned}
& -\Delta u^{n}+\nabla p^{n}=\lambda_{n} u^{n} \quad \text { in } \Omega, \\
& \operatorname{div} u^{n}=0 \quad \text { in } \Omega, \\
& u^{n}=0 \quad \text { on } \partial \Omega .
\end{aligned}
$$

For $t \in[0, T]$ set

$$
w^{n}(\xi, t)=\frac{1}{h(\xi, t)} A^{\varepsilon}(\xi, t) u^{n}(\xi)
$$

It is more convenient to work with the time-dependent sequence $\left\{w^{n}\right\}_{n=1}^{\infty}$ rather than $\left\{u^{n}\right\}_{n=1}^{\infty}$. Clearly $\left\{w^{n}(\cdot, t)\right\}_{n=1}^{\infty}$ are linearly independent and finite linear combinations from this set are dense in

$$
\left\{v \in H_{0}^{1}\left(\Omega ; \mathbb{R}^{3}\right): \operatorname{div}\left(h B^{\varepsilon}(\cdot, t) v\right)=0 \text { in } \Omega\right\}
$$

for each $t \in[0, T]$. A finite dimensional approximation $u^{\varepsilon N}$ is constructed as

$$
u^{\varepsilon N}(\xi, t)=\sum_{n=1}^{N} \phi_{n}(t) w^{n}(\xi, t),
$$

where $\phi_{n}:[0, T] \rightarrow \mathbb{R}$ are functions to be determined. Then, by construction, $\operatorname{div}\left(h B^{\varepsilon} u^{\varepsilon N}\right)=0$. Taking (3.6) into account we want $u^{\varepsilon N}$ to satisfy

$$
\int_{\Omega}\left(h D_{t} u^{\varepsilon N}-c D_{3} u^{\varepsilon N}\right) \cdot w^{m}+\nu h D u^{\varepsilon N} B^{\varepsilon}: D w^{m} B^{\varepsilon} \mathrm{d} \xi=\left\langle F_{\varepsilon}(t), w^{m}\right\rangle
$$

for $m=1, \ldots, N$ which is equivalent to

$$
C^{\varepsilon}(t) \phi^{\prime}(t)=D^{\varepsilon}(t) \phi(t)+f^{\varepsilon}(t),
$$

where $\phi=\left(\phi_{1}, \ldots, \phi_{N}\right), C^{\varepsilon}=\left\{c_{m n}^{\varepsilon}\right\}_{m, n=1}^{N}, D^{\varepsilon}=\left\{d_{m n}^{\varepsilon}\right\}_{m, n=1}^{N}$ and $f^{\varepsilon}=\left(f_{1}^{\varepsilon}, \ldots, f_{N}^{\varepsilon}\right)$ are given by

$$
\begin{aligned}
& c_{m n}^{\varepsilon}(t)=\int_{\Omega} h w^{n} \cdot w^{m} \mathrm{~d} \xi, \\
& d_{m n}^{\varepsilon}(t)=-\int_{\Omega}\left(h D_{t} w^{n}-c D_{3} w^{n}\right) \cdot w^{m}+\nu h D w^{n} B^{\varepsilon}: D w^{m} B^{\varepsilon} \mathrm{d} \xi, \\
& f_{m}^{\varepsilon}(t)=\left\langle F_{\varepsilon}(t), w^{m}\right\rangle .
\end{aligned}
$$


Note that $C^{\varepsilon}(t)$ is the Gram matrix of the linearly independent set $\left\{w^{n}(\cdot, t)\right\}_{n=1}^{N}$ with respect to the scalar product

$$
(u, v) \mapsto \int_{\Omega} u(\xi) \cdot v(\xi) h(\xi, t) \mathrm{d} \xi
$$

In particular $C^{\varepsilon}$ is invertible with bounded inverse and so (4.5) becomes

$$
\phi^{\prime}(t)=C^{\varepsilon}(t)^{-1} D^{\varepsilon}(t) \phi(t)+C^{\varepsilon}(t)^{-1} f^{\varepsilon}(t) .
$$

Next, we determine what initial condition to choose for $\phi$. The orthogonal projection

$$
P_{N}: L^{2}\left(\Omega ; \mathbb{R}^{3}\right) \rightarrow \operatorname{Span}\left\{w^{1}(\cdot, 0), \ldots, w^{N}(\cdot, 0)\right\}
$$

with respect to the scalar product (4.6) satisfies $P_{N}\left(u_{0}^{\varepsilon}\right)=\sum_{n=1}^{N} \alpha_{n} w^{n}(\cdot, 0)$ for some unique $\alpha=$ $\left(\alpha_{1}, \ldots, \alpha_{N}\right) \in \mathbb{R}^{N}$,

$$
\int_{\Omega}\left|P_{N}\left(u_{0}^{\varepsilon}\right)\right|^{2} h \mathrm{~d} \xi \leqslant \int_{\Omega}\left|u_{0}^{\varepsilon}\right|^{2} h \mathrm{~d} \xi \quad \text { and } \quad \lim _{N \rightarrow \infty} \int_{\Omega}\left|u_{0}^{\varepsilon}-P_{N}\left(u_{0}^{\varepsilon}\right)\right|^{2} h \mathrm{~d} \xi=0 .
$$

It is well known, see e.g. Filippov [5, Theorem 3, p. 5], that under the above stated conditions, the linear ODE (4.7) has a unique absolutely continuous solution $\phi:[0, T] \rightarrow \mathbb{R}^{N}$, satisfying $\phi(0)=\alpha$, or what is equivalent $u^{\varepsilon N}=P_{N}\left(u_{0}^{\varepsilon}\right)$ for $t=0$. This proves the existence of $u^{\varepsilon N}$ such that (4.4) holds.

From (4.4) it follows that

$$
\int_{\Omega}\left(h D_{t} u^{\varepsilon N}-c D_{3} u^{\varepsilon N}\right) \cdot u^{\varepsilon N}+\nu h\left|D u^{\varepsilon N} B^{\varepsilon}\right|^{2} \mathrm{~d} \xi=\left\langle F_{\varepsilon}(t), u^{\varepsilon N}\right\rangle .
$$

Using

$$
\frac{\mathrm{d}}{\mathrm{d} t} \int_{\Omega} \frac{1}{2} h\left|u^{\varepsilon N}\right|^{2} \mathrm{~d} \xi=\int_{\Omega} h D_{t} u^{\varepsilon N} \cdot u^{\varepsilon N}+\frac{1}{2} D_{t} h\left|u^{\varepsilon N}\right|^{2} \mathrm{~d} \xi
$$

and Lemmas 4.2 and 4.1 we obtain

$$
\begin{aligned}
\frac{\mathrm{d}}{\mathrm{d} t} \int_{\Omega} & \frac{1}{2}\left|u^{\varepsilon N}\right|^{2} h \mathrm{~d} \xi-\frac{1}{2} \int_{\Omega} D_{t} h\left|u^{\varepsilon N}\right|^{2} \mathrm{~d} \xi \\
& -K_{1}\left(\int_{\Omega}\left|D_{3} u^{\varepsilon N}\right|^{2} h \mathrm{~d} \xi\right)^{1 / 2}\left(\int_{\Omega}\left|u^{\varepsilon N}\right|^{2} h \mathrm{~d} \xi\right)^{1 / 2}+K_{2} \int_{\Omega}\left|D u^{\varepsilon N} E^{-1}\right|^{2} h \mathrm{~d} \xi \\
\leqslant & \left(\int_{\omega} \int_{h^{-}}^{h^{+}}\left|E D_{t} \hat{\mathcal{U}}\right|^{2} \mathrm{~d} z \mathrm{~d} x^{\prime}\right)^{1 / 2}\left(\int_{\Omega}\left|u^{\varepsilon N}\right|^{2} h \mathrm{~d} \xi\right)^{1 / 2} \\
& +\nu \sqrt{\lambda^{+}}\left(\int_{\omega} \int_{h^{-}}^{h^{+}}\left|E D \hat{\mathcal{U}} E^{-1}\right|^{2} \mathrm{~d} z \mathrm{~d} x^{\prime}\right)^{1 / 2}\left(\int_{\Omega}\left|D u^{\varepsilon N} E^{-1}\right|^{2} h \mathrm{~d} \xi\right)^{1 / 2} .
\end{aligned}
$$


Since

$$
\begin{aligned}
& \int_{\Omega} D_{t} h\left|u^{\varepsilon N}\right|^{2} \mathrm{~d} \xi \leqslant K_{3} \int_{\Omega}\left|u^{\varepsilon N}\right|^{2} h \mathrm{~d} \xi \\
& K_{1}\left(\int_{\Omega}\left|D_{3} u^{\varepsilon N}\right|^{2} h \mathrm{~d} \xi\right)^{1 / 2}\left(\int_{\Omega}\left|u^{\varepsilon N}\right|^{2} h \mathrm{~d} \xi\right)^{1 / 2} \\
& \quad \leqslant \frac{K_{2}}{4} \int_{\Omega}\left|D u^{\varepsilon N} E^{-1}\right|^{2} h \mathrm{~d} \xi+\varepsilon^{2} K_{4} \int_{\Omega}\left|u^{\varepsilon N}\right|^{2} h \mathrm{~d} \xi \\
& \nu \sqrt{\lambda^{+}}\left(\int_{\omega} \int_{h^{-}}^{h^{+}}\left|E D \hat{\mathcal{U}} E^{-1}\right|^{2} \mathrm{~d} z \mathrm{~d} x^{\prime}\right)^{1 / 2}\left(\int_{\Omega}\left|D u^{\varepsilon N} E^{-1}\right|^{2} h \mathrm{~d} \xi\right)^{1 / 2} \\
& \leqslant K_{5} \int_{\omega} \int_{h^{-}}^{h^{+}}\left|E D \hat{\mathcal{U}} E^{-1}\right|^{2} \mathrm{~d} z \mathrm{~d} x^{\prime}+\frac{K_{2}}{4} \int_{\Omega}\left|D u^{\varepsilon N} E^{-1}\right|^{2} h \mathrm{~d} \xi
\end{aligned}
$$

we deduce

$$
\begin{aligned}
& \frac{\mathrm{d}}{\mathrm{d} t} \int_{\Omega}\left|u^{\varepsilon N}\right|^{2} h \mathrm{~d} \xi+K_{2} \int_{\Omega}\left|D u^{\varepsilon N} E^{-1}\right|^{2} h \mathrm{~d} \xi \\
& \quad \leqslant \int_{\omega} \int_{h^{-}}^{h^{+}}\left|E D_{t} \hat{\mathcal{U}}\right|^{2} \mathrm{~d} z \mathrm{~d} x^{\prime}+K_{5} \int_{\omega} \int_{h^{-}}^{h^{+}}\left|E D \hat{\mathcal{U}} E^{-1}\right|^{2} \mathrm{~d} z \mathrm{~d} x^{\prime}+K_{6} \int_{\Omega}\left|u^{\varepsilon N}\right|^{2} h \mathrm{~d} \xi,
\end{aligned}
$$

where $K_{6}=1+K_{3}+2 \varepsilon_{0}^{2} K_{4}$. Applying Grönwall's inequality yields

$$
\sup _{0 \leqslant t \leqslant T} \int_{\Omega}\left|u^{\varepsilon N}\right|^{2} h \mathrm{~d} \xi \leqslant \int_{\Omega}\left|u_{0}^{\varepsilon}\right|^{2} h(\xi, 0) \mathrm{d} \xi+K_{7} R(\varepsilon),
$$

where

$$
R(\varepsilon)=\int_{0}^{T} \int_{\omega} \int_{h^{-}}^{h^{+}}\left|E D_{t} \hat{\mathcal{U}}\right|^{2}+\left|E D \hat{\mathcal{U}} E^{-1}\right|^{2} \mathrm{~d} z \mathrm{~d} x^{\prime} \mathrm{d} t .
$$

Integrating (4.8) from 0 to $T$ gives

$$
\int_{0}^{T} \int_{\Omega}\left|D u^{\varepsilon N} E^{-1}\right|^{2} h \mathrm{~d} \xi \mathrm{d} t \leqslant K_{8}\left(\varepsilon^{-1} \int_{\Omega_{\varepsilon}(0)}\left|\hat{u}_{0}^{\varepsilon}\right|^{2} \mathrm{~d} x+R(\varepsilon)\right) .
$$

From (2.18) it follows that $\varepsilon^{2} R(\varepsilon)$ is bounded for $0<\varepsilon \leqslant \varepsilon_{0}$. Taking also (2.24) into account, we deduce

$$
\begin{aligned}
& \left\|D_{3} u^{\varepsilon N}\right\|_{\Omega_{T}} \leqslant K \\
& \sup _{0 \leqslant t \leqslant T}\left\|u^{\varepsilon N}\right\|_{\Omega}+\left\|D_{1} u^{\varepsilon N}\right\|_{\Omega_{T}}+\left\|D_{2} u^{\varepsilon N}\right\|_{\Omega_{T}} \leqslant \varepsilon^{-1} K
\end{aligned}
$$


for some constant $K>0$. Owing to the Friedrichs inequality

$$
\left(\int_{\Omega}|v|^{2} \mathrm{~d} \xi\right)^{1 / 2} \leqslant 2\left(\int_{\Omega}\left|D_{3} v\right|^{2} \mathrm{~d} \xi\right)^{1 / 2}
$$

it holds also that $\left\|u^{\varepsilon N}\right\|_{\Omega_{T}} \leqslant 2 K$.

Using standard compactness and density arguments we obtain $u^{\varepsilon}$ as a weak subsequential limit of $\left\{u^{\varepsilon N}\right\}_{N=1}^{\infty}$. It is readily checked that $u^{\varepsilon}$ satisfies (3.6) and has all the properties stated in our definition of weak solution. Moreover $u^{\varepsilon}$ is unique and satisfies the estimates (4.1)-(4.2).

\section{Derivation of the Reynolds equation}

This section is devoted to the asymptotic analysis of $u^{\varepsilon}$, the solution of (3.6). Following [2], we introduce the scalar product

$$
(u, v) \mapsto \int_{\Omega} u \cdot v+D_{3} u \cdot D_{3} v \mathrm{~d} \xi
$$

and define $V_{\xi_{3}}$ as the completion of $C_{c}^{\infty}\left(\Omega ; \mathbb{R}^{3}\right)$ with respect to the norm induced by (5.1). Furthermore we denote as $\bar{f}$ the average in the $\xi_{3}$-direction of a function $f: \Omega \rightarrow \mathbb{R}^{k}$, i.e.

$$
\bar{f}\left(\xi^{\prime}\right)=\int_{0}^{1} f(\xi) \mathrm{d} \xi_{3}
$$

Lemma 5.1. Assume $F \in L^{2}\left(\Omega_{T} ; \mathbb{R}^{3}\right)$ with $\operatorname{div} F=0$ in $\Omega_{T}, F \cdot \hat{n}=g$ on $\Sigma^{w} \times(0, T)$, where $g \in L^{2}\left(\Sigma^{w} \times(0, T) ; \mathbb{R}^{3}\right)$. Then

$$
\frac{\mathrm{d}}{\mathrm{d} \xi_{3}} \int_{\omega_{T}} F_{3} \phi \mathrm{d} \xi^{\prime} \mathrm{d} t=\int_{\omega_{T}} F \cdot \nabla \phi \mathrm{d} \xi^{\prime} \mathrm{d} t-\int_{\partial \omega \times(0, T)} g \phi \mathrm{d} S\left(\xi^{\prime}\right) \mathrm{d} t
$$

for all $\phi \in L^{2}\left(0, T ; H^{1}(\omega)\right)$ and a.e. $\xi_{3} \in(0,1)$.

Proof. Assume $\phi \in L^{2}\left(0, T ; H^{1}(\omega)\right), \psi \in H_{0}^{1}(0,1)$ and set $\Phi(\xi, t)=\phi\left(\xi^{\prime}, t\right) \psi\left(\xi_{3}\right)$. Since $\operatorname{div}(\Phi F)=$ $\nabla \Phi \cdot F$, the Gauss-Green theorem implies

$$
\int_{\Omega} \nabla \Phi \cdot F \mathrm{~d} \xi=\int_{\Sigma^{w}} \Phi g \mathrm{~d} S(\xi) .
$$

Integration over $(0, T)$ gives

$$
\begin{aligned}
& \int_{0}^{1}\left(\int_{\omega_{T}} F_{3}(\xi, t) \phi\left(\xi^{\prime}, t\right) \mathrm{d} \xi^{\prime} \mathrm{d} t\right) \psi^{\prime} \mathrm{d} \xi_{3} \\
& \quad=\int_{0}^{1}\left(-\int_{\omega_{T}} F(\xi, t) \cdot \nabla \phi\left(\xi^{\prime}, t\right) \mathrm{d} \xi^{\prime} \mathrm{d} t+\int_{\partial \omega \times(0, T)} g(\xi, t) \phi\left(\xi^{\prime}, t\right) \mathrm{d} S\left(\xi^{\prime}\right) \mathrm{d} t\right) \psi \mathrm{d} \xi_{3} .
\end{aligned}
$$

This proves (5.2). 
Lemma 5.2. Let $u^{\varepsilon}$ be the sequence of solutions of (3.6). Then there exists $u^{*} \in L^{2}\left(0, T ; V_{\xi_{3}}\right)$ such that up to a subsequence $u^{\varepsilon} \rightarrow u^{*}$ in $L^{2}\left(0, T ; V_{\xi_{3}}\right)$ as $\varepsilon \rightarrow 0$. Moreover,

(1) $u^{*}(\xi, t)=0$ on $\Sigma^{ \pm}$for a.e. $t \in[0, T]$.

(2) $u_{3}^{*}=0$ a.e. in $\Omega \times(0, T)$.

(3) $\operatorname{div} h \overline{u^{*}}=0$ in $\omega \times(0, T)$ and $h \overline{u^{*}} \cdot \hat{n}=0$ on $\partial \omega \times(0, T)$.

Proof. By (4.1), $u^{\varepsilon}$ is bounded in the Hilbert space $L^{2}\left(0, T ; V_{\xi_{3}}\right)$. Thus there exists a subsequence such that $u^{\varepsilon} \rightarrow u^{*}$ in $L^{2}\left(0, T ; V_{\xi_{3}}\right)$. The inequality

$$
\int_{\Sigma^{-} \cup \Sigma^{+}}|v|^{2} \mathrm{~d} S \leqslant(1+\sqrt{2}) \int_{\Omega}|v|^{2}+\left|D_{3} v\right|^{2} \mathrm{~d} \xi
$$

which holds for all $v \in C^{1}\left(\bar{\Omega} ; \mathbb{R}^{3}\right)$ implies that $u^{*}=0$ on $\Sigma^{-} \cup \Sigma^{+}$in the trace sense.

Passing to the limit in

$$
\int_{\Omega_{T}} \varepsilon h B^{\varepsilon} u^{\varepsilon} \cdot \nabla v \mathrm{~d} \xi \mathrm{d} t=0
$$

gives

$$
\int_{\Omega_{T}} u_{3}^{*} D_{3} v \mathrm{~d} \xi \mathrm{d} t=0
$$

for all $v \in L^{2}\left(0, T ; H^{1}(\Omega)\right)$. Thus $D_{3} u_{3}^{*}=0$ and so Friedrichs' inequality (4.12) implies $u_{3}^{*}=0$ in $\Omega \times(0, T)$. In view of (5.4) and Lemma 5.1, with $F^{\varepsilon}=h B^{\varepsilon} u^{\varepsilon}$,

$$
\int_{\omega_{T}}\left(F_{3}^{\varepsilon}\left(\xi^{\prime}, b, t\right)-F_{3}^{\varepsilon}\left(\xi^{\prime}, a, t\right)\right) \phi\left(\xi^{\prime}, t\right) \mathrm{d} \xi^{\prime} \mathrm{d} t=\int_{a}^{b}\left(\int_{\omega_{T}} F^{\varepsilon}(\xi, t) \cdot \nabla \phi\left(\xi^{\prime}, t\right) \mathrm{d} \xi^{\prime} \mathrm{d} t\right) \mathrm{d} \xi_{3}
$$

for all $\phi \in L^{2}\left(0, T ; H^{1}(\omega)\right)$ and $a, b \in(0,1)$. Since $F_{3}^{\varepsilon}=0$ on $\Sigma^{ \pm}$we conclude that

$$
\begin{aligned}
0 & =\lim _{\varepsilon \rightarrow 0} \int_{0}^{1}\left(\int_{\omega_{T}} F^{\varepsilon}(\xi, t) \cdot \nabla \phi\left(\xi^{\prime}, t\right) \mathrm{d} \xi \mathrm{d} t\right) \mathrm{d} \xi_{3} \\
& =\int_{\omega_{T}}\left(\int_{0}^{1} h\left(\xi^{\prime}, t\right) u^{*}(\xi, t) \mathrm{d} \xi_{3}\right) \cdot \nabla \phi\left(\xi^{\prime}, t\right) \mathrm{d} \xi^{\prime} \mathrm{d} t
\end{aligned}
$$

for all $\phi \in L^{2}\left(0, T ; H^{1}(\omega)\right)$, that is $\operatorname{div} h \overline{u^{*}}=0$ in $\omega_{T}$ and $h \overline{u^{*}} \cdot \hat{n}=0$ on $\partial \omega \times(0, T)$.

At this point it is convenient to introduce

$$
\mathcal{U}(\xi, t)=\hat{\mathcal{U}}\left(E^{-1} \psi^{\varepsilon}(\xi, t), t\right)=\hat{\mathcal{U}}\left(\xi_{1}, \xi_{2},\left(1-\xi_{3}\right) h^{-}+\xi_{3} h^{+}, t\right) .
$$


Observe that

$$
\begin{aligned}
& \operatorname{div}(h B \mathcal{U})=0 \quad \text { in } \Omega, \\
& \mathcal{U}=\left(v^{ \pm}, D_{t} h^{ \pm}+v^{ \pm} \cdot \nabla h^{ \pm}\right) \quad \text { on } \Sigma^{ \pm}, \\
& \mathcal{U}=g \quad \text { on } \Sigma^{w}=\partial \omega \times(0,1),
\end{aligned}
$$

where $g(\xi, t)=\hat{g}\left(E^{-1} \psi^{\varepsilon}(\xi, t), t\right)$.

Lemma 5.3. Assume, as in Lemma 5.2, that $u^{*}$ is a weak subsequential limit of $u^{\varepsilon}$. Then there exists $p^{*} \in L^{2}\left(0, T ; \stackrel{\circ}{L}(\omega) \cap H^{1}(\omega)\right)$ such that

$$
u^{*}=-\frac{\xi_{3}\left(1-\xi_{3}\right) h^{2}}{2 \nu} \nabla p^{*}+\xi_{3} v^{+}+\left(1-\xi_{3}\right) v^{-}-P \mathcal{U},
$$

where

$$
\stackrel{\circ}{L}^{2}(\omega)=\left\{p \in L^{2}(\omega): \int_{\omega} p \mathrm{~d} \xi^{\prime}=0\right\}
$$

and $P$ denotes the projection $P y=\left(y_{1}, y_{2}, 0\right), y \in \mathbb{R}^{3}$.

Proof. As a first step it is shown that

$$
U^{*}=\frac{\nu}{h^{2}} P\left(u^{*}+\mathcal{U}\right)
$$

satisfies the so called thin-film equation

$$
D_{3}^{2} U^{*}=\nabla p^{*}
$$

To this end, multiply (3.6) with $\psi \in C^{1}([0, T])$ such that $\psi(T)=0$ and integrate (by parts) over $[0, T]$. Thus we obtain

$$
\begin{aligned}
& -\psi(0) \int_{\Omega} u_{0}^{\varepsilon} \cdot A^{\varepsilon} v \mathrm{~d} \xi-\int_{0}^{T} \psi^{\prime}(t) \int_{\Omega} u^{\varepsilon} \cdot A^{\varepsilon} v \mathrm{~d} \xi \mathrm{d} t \\
& \quad+\int_{0}^{T} \psi(t) \int_{\Omega}-u^{\varepsilon} \cdot D_{t} A^{\varepsilon} v-\frac{c}{h} D_{3} u^{\varepsilon} \cdot A^{\varepsilon} v+\nu h D u^{\varepsilon} B^{\varepsilon}: D\left(A^{\varepsilon} v / h\right) B^{\varepsilon} \mathrm{d} \xi \mathrm{d} t \\
& =\int_{0}^{T} \psi(t)\left\langle F^{\varepsilon}(t), A^{\varepsilon} v / h\right\rangle \mathrm{d} t .
\end{aligned}
$$

Next, multiply the above equation with $\varepsilon^{2}$ and let $\varepsilon \rightarrow 0$. From (4.1) it is easily seen that the first four terms on the left side tend to zero. As to the fifth term, we write

$$
D u^{\varepsilon} B^{\varepsilon}: D\left(A^{\varepsilon} v / h\right) B^{\varepsilon}=\left(E D u^{\varepsilon} E^{-2}\right)\left(E^{2} B E^{-2}\right): D(A v / h) B
$$


and observe that

$$
\varepsilon^{2} E D u^{\varepsilon} E^{-2}=\left(\begin{array}{ccc}
\varepsilon^{2} D_{1} u_{1}^{\varepsilon} & \varepsilon^{2} D_{2} u_{1}^{\varepsilon} & D_{3} u_{1}^{\varepsilon} \\
\varepsilon^{2} D_{1} u_{2}^{\varepsilon} & \varepsilon^{2} D_{2} u_{2}^{\varepsilon} & D_{3} u_{2}^{\varepsilon} \\
\varepsilon^{3} D_{1} u_{2}^{\varepsilon} & \varepsilon^{3} D_{2} u_{2}^{\varepsilon} & \varepsilon D_{3} u_{2}^{\varepsilon}
\end{array}\right) \rightarrow\left(\begin{array}{ccc}
0 & 0 & D_{3} u_{1}^{*} \\
0 & 0 & D_{3} u_{2}^{*} \\
0 & 0 & 0
\end{array}\right)
$$

weakly in $L^{2}\left(\Omega_{T}\right)$, while

$$
E^{2} B E^{-2}=\left(\begin{array}{ccc}
1 & 0 & 0 \\
0 & 1 & 0 \\
-\varepsilon^{2} a / h & -\varepsilon^{2} b / h & 1 / h
\end{array}\right) \rightarrow\left(\begin{array}{ccc}
1 & 0 & 0 \\
0 & 1 & 0 \\
0 & 0 & 1 / h
\end{array}\right)
$$

uniformly on $\Omega_{T}$. Thus

$$
\begin{aligned}
\lim _{\varepsilon \rightarrow 0} \varepsilon^{2} \int_{0}^{T} \psi(t) \int_{\Omega} \nu h D u^{\varepsilon} B^{\varepsilon}: D\left(A^{\varepsilon} v / h\right) B^{\varepsilon} \mathrm{d} \xi \mathrm{d} t \\
\quad=\int_{0}^{T} \psi(t) \int_{\Omega} \nu h\left(\begin{array}{ccc}
0 & 0 & D_{3} u_{1}^{*} / h \\
0 & 0 & D_{3} u_{2}^{*} / h \\
0 & 0 & 0
\end{array}\right): D(A v / h) B \mathrm{~d} \xi \mathrm{d} t \\
\quad=\int_{0}^{T} \psi(t) \int_{\Omega} \frac{\nu}{h^{2}}\left(D_{3} u_{1}^{*} D_{3} v_{1}+D_{3} u_{2}^{*} D_{3} v_{2}\right) \mathrm{d} \xi \mathrm{d} t .
\end{aligned}
$$

Similarly one finds that

$$
\begin{aligned}
& \lim _{\varepsilon \rightarrow 0} \varepsilon^{2} \int_{0}^{T} \psi(t)\left\langle F^{\varepsilon}(t), A^{\varepsilon} v / h\right\rangle \mathrm{d} t \\
& \quad=-\int_{0}^{T} \psi(t) \int_{\Omega} \frac{\nu}{h^{2}}\left(D_{3} \mathcal{U}_{1}^{*} D_{3} v_{1}+D_{3} \mathcal{U}_{2}^{*} D_{3} v_{2}\right) \mathrm{d} \xi \mathrm{d} t .
\end{aligned}
$$

Thus one obtains

$$
\begin{aligned}
0 & =\int_{0}^{T} \psi(t) \int_{\Omega} \frac{\nu}{h^{2}} D_{3} P\left(u^{*}+\mathcal{U}\right) \cdot D_{3} v \mathrm{~d} \xi \mathrm{d} t \\
& =\int_{0}^{T} \psi(t) \int_{\Omega} D_{3} U^{*} \cdot D_{3} v \mathrm{~d} \xi \mathrm{d} t
\end{aligned}
$$

for all $v \in V$ and all $\psi \in C^{1}([0, T])$ such that $\psi(T)=0$. In view of de Rham's theorem, see e.g. [8], there exists $p^{*} \in L^{2}(0, T ; \stackrel{\circ}{L}(\Omega))$ such that

$$
\int_{\Omega_{T}} D_{3} U^{*} \cdot D_{3} P v \mathrm{~d} \xi \mathrm{d} t=\int_{\Omega_{T}} p^{*} \operatorname{div} v \mathrm{~d} \xi \mathrm{d} t
$$

for all $v \in L^{2}\left(0, T ; H_{0}^{1}\left(\Omega ; \mathbb{R}^{3}\right)\right)$, i.e. $U^{*}$ satisfies (5.11) in a weak sense. Observe that (5.12) implies $D_{3} p^{*}=0$ in $\Omega_{T}$, hence $p^{*}(\xi, t)=p^{*}\left(\xi^{\prime}, t\right)$, i.e. $p^{*}$ does not depend on $\xi_{3}$. It remains to show that 
$p^{*} \in L^{2}\left(0, T ; H^{1}(\omega)\right)$. To this end choose $v(\xi)=\psi\left(\xi_{3}\right) \phi\left(\xi^{\prime}\right)$ in (5.12), where $\phi \in H_{0}^{1}\left(\omega ; \mathbb{R}^{2}\right)$ and $\psi \in H_{0}^{1}(0,1)$. Using Fubini's theorem we obtain

$$
\int_{0}^{1}\left(\int_{\omega_{T}} D_{3} U^{*} \cdot \phi \mathrm{d} \xi^{\prime} \mathrm{d} t\right) \psi^{\prime} \mathrm{d} \xi_{3}=\int_{0}^{1}\left(\int_{\omega_{T}} p^{*} \operatorname{div} \phi \mathrm{d} \xi^{\prime} \mathrm{d} t\right) \psi \mathrm{d} \xi_{3} .
$$

Let the function $z:(0,1) \rightarrow \mathbb{R}$ and the constant $\lambda \in \mathbb{R}$ be defined by

$$
\begin{aligned}
& z\left(\xi_{3}\right)=\int_{\omega_{T}} U^{*}(\xi, t) \cdot \phi\left(\xi^{\prime}, t\right) \mathrm{d} \xi^{\prime} \mathrm{d} t \\
& \lambda=\int_{\omega_{T}} p^{*}\left(\xi^{\prime}, t\right) \operatorname{div} \phi\left(\xi^{\prime}, t\right) \mathrm{d} \xi^{\prime} \mathrm{d} t .
\end{aligned}
$$

Then $z \in H^{1}(0,1)$ and (5.13) says that

$$
-z^{\prime \prime}=\lambda \quad \text { in }(0,1)
$$

The general solution of $(5.14)$ is

$$
z\left(\xi_{3}\right)=\frac{\lambda}{2} \xi_{3}\left(1-\xi_{3}\right)+z(1) \xi_{3}+z(0)\left(1-\xi_{3}\right)
$$

Taking into account that $U^{*}=\nu h^{-2} v^{ \pm}$on $\Sigma^{ \pm}$, we obtain

$$
\int_{\omega_{T}} U^{*} \cdot \phi \mathrm{d} \xi^{\prime} \mathrm{d} t=\int_{\omega_{T}} \frac{1}{2} \xi_{3}\left(1-\xi_{3}\right) p^{*} \operatorname{div} \phi+\frac{\nu}{h^{2}}\left(\xi_{3} v^{+}+\left(1-\xi_{3}\right) v^{-}\right) \cdot \phi \mathrm{d} \xi^{\prime} \mathrm{d} t .
$$

Integrating this equality from $\xi_{3}=0$ to 1 using Fubini gives

$$
\int_{\omega_{T}}\left(-12 \overline{U^{*}}+\frac{6 \nu}{h^{2}}\left(v^{+}+v^{-}\right)\right) \cdot \phi \mathrm{d} \xi^{\prime} \mathrm{d} t=\int_{\omega_{T}} p^{*} \operatorname{div} \phi \mathrm{d} \xi^{\prime} \mathrm{d} t .
$$

This shows that $p^{*} \in L^{2}\left(0, T ; H^{1}(\omega)\right)$ with

$$
\nabla p^{*}=12 \overline{U^{*}}-\frac{6 \nu}{h^{2}}\left(v^{+}+v^{-}\right) .
$$

On integrating by parts in (5.16), we deduce that

$$
U^{*}=-\frac{1}{2} \xi_{3}\left(1-\xi_{3}\right) \nabla p^{*}+\frac{\nu}{h^{2}}\left(\xi_{3} v^{+}+\left(1-\xi_{3}\right) v^{-}\right) .
$$

From this (5.10) follows. 
Theorem 5.1. As $\varepsilon \rightarrow 0$, the whole sequence $u^{\varepsilon}$ of solutions of (3.6) converges weakly in $L^{2}\left(0, T ; V_{\xi_{3}}\right)$ to

$$
u^{*}=-\frac{\xi_{3}\left(1-\xi_{3}\right) h^{2}}{2 \nu} \nabla p^{*}+\xi_{3} v^{+}+\left(1-\xi_{3}\right) v^{-}-P \mathcal{U}
$$

where $p^{*} \in L^{2}\left(0, T ; \stackrel{\circ}{L}^{2}(\omega) \cap H^{1}(\omega)\right)$ is the unique solution of the Reynolds equation

$$
\begin{cases}D_{t} h+\operatorname{div}\left(-\frac{h^{3}}{12 \nu} \nabla p^{*}+\frac{h}{2}\left(v^{+}+v^{-}\right)\right)=0 & \text { in } \omega \times(0, T), \\ \left(-\frac{h^{3}}{12 \nu} \nabla p^{*}+\frac{h}{2}\left(v^{+}+v^{-}\right)\right) \cdot \hat{n}=h \bar{g} \cdot \hat{n} \quad & \text { on } \partial \omega \times(0, T),\end{cases}
$$

where

$$
\bar{g}\left(x^{\prime}, t\right)=\int_{h^{-}\left(x^{\prime}, t\right)}^{h^{+}\left(x^{\prime}, t\right)} \hat{g}\left(x^{\prime}, z, t\right) \mathrm{d} z .
$$

Proof. Equation (5.10) implies

$$
h \overline{u^{*}}+h \overline{P \mathcal{U}}=-\frac{h^{3}}{12 \nu} \nabla p^{*}+\frac{h}{2}\left(v^{+}+v^{-}\right) .
$$

Applying Lemma 5.1 with $F=h B \mathcal{U}$ yields

$$
\begin{aligned}
\int_{\omega_{T}} h \overline{P \mathcal{U}} \cdot \nabla \phi \mathrm{d} \xi^{\prime} \mathrm{d} t= & \int_{0}^{1}\left(\int_{\omega_{T}} F \cdot \nabla \phi \mathrm{d} \xi^{\prime} \mathrm{d} t\right) \mathrm{d} \xi_{3} \\
= & \int_{\omega_{T}}\left(F_{3}\left(\xi^{\prime}, 1, t\right)-F_{3}\left(\xi^{\prime}, 0, t\right)\right) \phi \mathrm{d} \xi^{\prime} \mathrm{d} t \\
& +\int_{0}^{1}\left(\int_{\partial \omega \times(0, T)} \phi F \cdot \hat{n} \mathrm{~d} S\left(\xi^{\prime}\right) \mathrm{d} t\right) \mathrm{d} \xi_{3} \\
= & \int_{\omega_{T}} D_{t} h \phi \mathrm{d} \xi^{\prime} \mathrm{d} t+\int_{\partial \omega \times(0, T)} \phi h \bar{g} \cdot \hat{n} \mathrm{~d} S\left(\xi^{\prime}\right) \mathrm{d} t .
\end{aligned}
$$

Combining this with Lemma 5.2(3) we deduce

$$
\begin{aligned}
& \int_{\omega_{T}}\left(-\frac{h^{3}}{12 \nu} \nabla p^{*}+\frac{h}{2}\left(v^{+}+v^{-}\right)\right) \cdot \nabla \phi \mathrm{d} \xi^{\prime} \mathrm{d} t \\
& =\int_{\omega_{T}} D_{t} h \phi \mathrm{d} \xi^{\prime} \mathrm{d} t+\int_{\partial \omega \times(0, T)} \phi h \bar{g} \cdot \hat{n} \mathrm{~d} S\left(\xi^{\prime}\right) \mathrm{d} t
\end{aligned}
$$


for all $\phi \in L^{2}\left(0, T ; H^{1}(\omega)\right)$. The integral identity (5.21) is indeed the weak formulation of (5.20). Furthermore (5.21) has a unique solution if and only if

$$
\int_{0}^{T}\left(\int_{\omega} D_{t} h \mathrm{~d} \xi^{\prime}+\int_{\partial \omega} h \bar{g} \cdot \hat{n} \mathrm{~d} S\left(\xi^{\prime}\right)\right) \phi \mathrm{d} t=0
$$

for all $\phi \in L^{2}(0, T)$. Since this condition is equivalent to assumption (2.9), we conclude that $p^{*}$ is uniquely determined by (5.21). Hence the whole sequence $u^{\varepsilon}$ converges to $u^{*}$.

\section{Concluding remarks}

Summing up, we have proved weak convergence of the "velocity field" $u^{\varepsilon}$ and found an expression for the limit $u^{*}$, see Theorem 5.1. Unlike previous studies, no estimates for the pressure $\left(P^{\varepsilon}\right.$ in $\left.(2.1)\right)$ were used to pass to the limit. In fact we have not even showed the existence of such a function, although it can be deduced from (3.6) and de Rham's theorem. Nevertheless a "limit pressure" $p^{*}$ appears in the Reynolds equation (5.20). The reason for this is that de Rham's theorem was invoked only after letting $\varepsilon \rightarrow 0$. The authors hope that convergence of the pressure can be addressed in future work.

\section{Acknowledgements}

This work was supported by Bernt Järmarks Foundation for scientific Research, by RFBR (project 12-01-00214), by a Government grant of the Russian Federation under the Resolution No. 220 "On measures designed to attract leading scientists to Russian institutions of higher learning" under agreement No. 11.G34.31.0054 and by a grant from the Swedish Research Council (project 621-2008-5186).

\section{References}

[1] G. Bayada and M. Chambat, The transition between the Stokes equation and the Reynolds equation: a mathematical proof, Appl. Math. Optim. 14(1) (1986), 73-93.

[2] G. Bayada, M. Chambat and I. Ciuperca, Asymptotic Navier-Stokes equations in a thin moving boundary domain, Asymptot. Anal. 21(2) (1999), 117-132.

[3] G. Cimatti, How the Reynolds equation is related to the Stokes equations, Appl. Math. Optim. 10(3) (1983), $267-274$.

[4] J. Fabricius, Homogenization of some problems in hydrodynamic lubrication involving rough boundaries, PhD thesis, Luleå University of Technology, 2011.

[5] A.F. Filippov, Differential Equations with Discontinuous Righthand Sides, Kluwer Academic Publishers, Dordrecht, 1988.

[6] S. Marušić and E. Marušić-Paloka, Two-scale convergence for thin domains and its applications to some lower-dimensional models in fluid mechanics, Asymptot. Anal. 23(1) (2000), 23-57.

[7] O. Reynolds, On the theory of lubrication and its application to Mr. Beauchamp Tower's experiments, including an experimental determination of the viscosity of olive oil, Philosophical Transactions of the Royal Society of London 177 (1886), $157-234$.

[8] R. Temam, Navier-Stokes Equations: Theory and Numerical Analysis, North-Holland, Amsterdam, 1979.

[9] J. Wilkening, Practical error estimates for Reynolds' lubrication approximation and its higher order corrections, SIAM J. Math. Anal. 41(2) (2009), 588-630. 